\section{Re: Quality of Life Associated with Daily Opioid Therapy in a Primary Care Chronic Pain Sample}

To the Editor: I read with interest the recent article by Dillie and colleagues ${ }^{1}$ in the March-April issue of the Fournal of the American Board of Family Medicine but was disappointed that the authors failed to point out several obvious limitations to their conclusions. The authors concluded that patients in the $20 \mathrm{mg}$ to $40 \mathrm{mg} / \mathrm{d}$ morphine equivalent group had significantly better Health Related Quality of Life (HRQoL) scores than their nonopioid treated or higher dosed counterparts. But what is still unknown, and not answered by this evaluation, is to what degree the prescribed opioid, assuming the patient is taking what is prescribed, has already improved those patients' HRQoL since the initiation of opioid therapy? The authors note that a more appropriate comparison, but not very feasible, would have been to compare groups' HRQoL with and without opioid therapy; but this was not done. Therefore, it is inaccurate to conclude that patients who were prescribed "high dose" opioids had lower HRQoL solely because of the total daily dose of their prescribed opioid.

The authors state that their findings "support recent recommendations that primary care physicians limit opioid dosing to $<100 \mathrm{mg} / \mathrm{d}$ " and provide 3 references to support this conclusion. However, this conclusion is not supported by any of the cited references, nor is it supported by this or any other published study. This recommendation is also suspect because there is no mention by these authors, or any of the cited supporting studies, as to which opioid this recommendation this applies to. If not corrected, this study will only add to the confusion surrounding the appropriate use of opioids in non-cancer patients who experience pain rather than finding any meaningful solution for those in the greatest need.

David S. Craig, PharmD, BCPS

H. Lee Moffitt Cancer Center and Research Institute Tampa, FL david.craig@moffitt.org

\section{Reference}

1. Dillie KS, Fleming MF, Mundt MP, French MT. Quality of life associated with daily opioid therapy in a primary care chronic pain sample. J Am Board Fam Med 2008;21:108-17.

doi: 10.3122/jabfm.2008.05.080097

Dr. Fleming declined to respond to this letter.

\section{Re: The Association Between Hay Fever and Stroke in a Cohort of Middle Aged and Elderly Adults}

To the Editor: We read with interest the paper by Matheson et $\mathrm{al}^{1}$ on the increased risk of stroke in those with hey fever (allergic rhinitis). The authors suggested the mechanism to be the systemic inflammation found in those with allergic rhinitis. We would suggest an additional explanation, ie, obstructive sleep apnea (OSA), for the increased risk.

Allergic rhinitis was associated with increased risk of OSA in adults ${ }^{2}$ and children. ${ }^{3}$ Nasal blockage caused by allergic rhinitis would lead to mouth breathing. Chronic mouth breathing will predispose to a steep mandibular plane that results in a narrow upper airway. A narrow upper airway would predispose to OSA. OSA with apnea-induced hypoxia and exaggerated negative intrathoracic pressure, arousals from sleep, surges in blood pressure, alteration in autonomic nervous activity, as well as production of reactive oxygen species, vascular endothelial growth factor, and inflammatory mediators are factors contributing to the vascular consequences. OSA is associated with atherosclerosis in adults. ${ }^{4}$ Adults with OSA had an increased risk of cerebrovascular disease independent of atherosclerotic risk factors. ${ }^{5}$

Recent researches of OSA found that inflammation was involved in the process. ${ }^{6} \mathrm{C}$-reactive protein (CRP) had been shown to increase even in children with OSA. CRP dropped after tonsillo-adenoidectomy in children. ${ }^{7}$ Other factors that might be involved in atherosclerosis, like fibrinogen level and p-selectin (marker of platelet activation), were shown to be higher in those with OSA.

Another potentially important factor is excessive daytime sleepiness. Allergic rhinitis ${ }^{8}$ was strongly associated with excessive daytime sleepiness (EDS). EDS is a well known risk factor for stroke in middle age and elderly. Previous studies showed that EDS, even after adjusted for the effect of sleep disordered breathing, is associated with stroke. ${ }^{9}$

In conclusion, allergic rhinitis is a very common condition that is associated with OSA that carries a high risk of mortality and morbidities. Appropriate treatment of childhood allergic rhinitis may prevent the development of OSA by preventing chronic oral mouth breathing. Adults with allergic rhinitis and habitual snoring should undergo a sleep polysomnography study as to allow diagnosis and treatment of OSA. 